\title{
Major Maternal Dietary Patterns during Early Pregnancy and Their Association with Neonatal Anthropometric Measurement
}

\author{
Hossein Hajianfar, ${ }^{1,2,3}$ Ahmad Esmaillzadeh, ${ }^{1,4}$ Awat Feizi, ${ }^{5}$ \\ Zahra Shahshahan, ${ }^{6}$ and Leila Azadbakht ${ }^{10}{ }^{1,2,4}$ \\ ${ }^{1}$ Food Security Research Center, Isfahan University of Medical Sciences, Isfahan, Iran \\ ${ }^{2}$ Research Committee of Community Nutrition, School of Nutrition and Food Sciences, \\ Isfahan University of Medical Sciences, Isfahan, Iran \\ ${ }^{3}$ Department of Nutrition, School of Nutrition and Food Sciences, Semnan University of Medical Sciences, Semnan, Iran \\ ${ }^{4}$ Department of Community Nutrition, School of Nutritional Sciences and Dietetics, \\ Tehran University of Medical Sciences, Tehran, Iran \\ ${ }^{5}$ Departments of Biostatistics and Epidemiology, School of Health, Isfahan University of Medical Sciences, Isfahan, Iran \\ ${ }^{6}$ Department of Gynecology, School of Medicine, Isfahan University of Medical Sciences, Isfahan, Iran
}

Correspondence should be addressed to Leila Azadbakht; azadbakhtleila@gmail.com

Received 15 December 2017; Revised 23 February 2018; Accepted 18 March 2018; Published 31 May 2018

Academic Editor: Ricardo E. Fretes

Copyright (C) 2018 Hossein Hajianfar et al. This is an open access article distributed under the Creative Commons Attribution License, which permits unrestricted use, distribution, and reproduction in any medium, provided the original work is properly cited.

Background. Anthropometric measurements of newborn infant are widely assessed as determinants of maternal nutrition. Although earlier studies have mostly examined the effects of particular nutrients or foods during gestational period on neonatal anthropometric measurements, there are few studies regarding the association of dietary patterns and mentioned measurements. So, the purpose of the current study was to investigate the association between major maternal dietary patterns and neonatal anthropometric measurements including body weight, head circumference, and height. Methods. The current prospective observational study is based on the data collected from 812 pregnant women. Dietary data was collected using a validated semiquantitative food frequency questionnaire. Results. Three identified major dietary patterns according to the results obtained from the factor loading matrix were (i) "western dietary pattern"; (ii) "traditional dietary pattern"; (iii) "healthy dietary pattern". Overall, this study demonstrated a positive significant association between high adherences to western dietary pattern and chance of having low birth weight infant. However, such associations were not seen in women taking healthy and traditional dietary patterns. Conclusion. We found that healthier maternal dietary patterns during early pregnancy might be associated with lower risk of low birth weight. Further studies are required to confirm these findings.

\section{Introduction}

Anthropometric measurements, including body weight, head circumference, and height of newborn infants, are widely assessed as determinants of impaired fetal growth, intrauterine environment, and maternal nutrition [1]. Restricted fetal growth is one of the most important global public health problems which provides a foundation for developing chronic diseases throughout their life [2]. Low birth weight infants are susceptible to higher risk of developing iron deficiency anemia leading to impaired development and altered longer term neurodevelopment [3]. Also, impaired fetal growth, in particular in head circumference, is associated with nonoptimal neurodevelopmental outcome [4]. There are also several evidences regarding the association between the impaired growth indices at birth and increased risk of developing some chronic disorders such as obesity, diabetes [5], cardiovascular diseases [5], endothelial dysfunction [6], nonalcoholic fatty liver disease [7], and chronic kidney disease [8].

In addition to genetic factors [9], placenta structural [10] and environmental factors affect fetal growth in utero [9], and maternal nutritional status could impact on fetal growth and 
development [11]. There is increasing evidence that nutrients $[12]$ and some foods $[13,14]$ play an important role in the fetal growth. Earlier studies have also shown that high-quality diet in the first trimester of pregnancy is positively associated with birth size including birth weight and birth length [15].

However, due to a wide range of nutrient interactions, maternal dietary patterns should be explored to achieve the association between maternal nutrition and fetal growth. It is noteworthy that there are few studies assessing the birth anthropometric measurements along the maternal dietary patterns [16].

Dietary patterns are different based on cultural, geographical, and regional influence in each area and can have effects on health outcomes $[17,18]$. Limited data are available regarding the association of maternal dietary patterns and neonatal anthropometric measurements in Middle Eastern countries, where mentioned factors are different from western population [19].

So, the purpose of the current study was to investigate the association between major maternal dietary patterns during the first-trimester period and neonatal anthropometric measurements including body weight, head circumference, and height. Such findings will be used to implement informative interventional programs to control impaired fetal growth and develop practical policies to improve the diet quality among pregnant women.

\section{Methods}

2.1. Study Design and Participants. The current prospective observational study was conducted among pregnant women during the first trimester, who were being attended at health centers across Isfahan city in the central part of Iran during 2015-2016. A random sample of 812 pregnant women was selected from 20 various health centers by the multistage cluster random sampling method. Eligible criteria included singleton pregnant women during the first trimester without any medical condition, use of medications, and without following a specific diet. Exclusion criteria were avoiding of follow-up during the study and current-smoker women. Also we exclude twin pregnancies.

Informed consent was obtained from all participants. This study was approved by the research council (research project number: 193053) and ethics committee (research ethics number: IR.MUI.REC193053).

\subsection{Data Collection}

2.2.1. Assessment of Dietary Intake. Pregnant women in these analyses completed validated 117-item semiquantitative food frequency questionnaire (FFQ) in the first visit, at 8-16 weeks. The validity and reliability of FFQ had been previously evaluated [20]. We inquired about the consumption of each food item, based on commonly used units or portion sizes, over the preceding 12 months on a daily, weekly, or monthly basis. Participants were asked to report their dietary intakes of foods based on nine multiple choice frequency response categories varying from "never or less than once a month" to " 12 or more times per day". Portion sizes of consumed foods were converted to grams from household measures. Supplements were also included to assess total nutrient intake. Then nutrient and energy intakes were computed by using NUTRITIONIST IV software (version 7.0; N-Squared Computing, Salem, OR), which was designed for Iranian foods. All nutrient values were energy-adjusted using the residuals method.

2.2.2. Determination of Dietary Patterns. We applied principal component analysis in order to find major dietary patterns. Food items similar in nutrient profile were combined into 33 predefined food groups (Table 1). When the item was unique in the nutrient profile, it was not combined (e.g., salt). The factors were rotated by varimax rotation function. Correlated variables are aggregated by factor analysis. The obtained factors are linear combinations of the included variables, explaining as much variation in the original variables as possible. Three factors were retained, based on the screen plot, an eigenvalue of more than 1.9, and the interpretability of the derived factors and of the earlier literature.

Based on our interpretation of the data and of the earlier literature, these 3 factors were labeled as the healthy, western, and traditional patterns. The individual scores for the 3 patterns show the values estimated for each individual based on their consumption of foods and the factor loadings of the foods.

2.2.3. Assessment of Other Variables. For the infants, birth date, gestational age, and anthropometric measurements including body weight, head circumference, and height were recorded at birth. Neonatal anthropometric measurements were categorized according to $\mathrm{WHO}$ standards and as follows: low birth weight (LBW); a birth weight less than 2500 g, normal birth weight; a birth weight more than $2500 \mathrm{~g}$ and less than 3900, low height; a height less than $47 \mathrm{~cm}$, normal height; a height more than $47 \mathrm{~cm}$ and less than $55 \mathrm{~cm}$, low head circumference; a head circumference less than $33 \mathrm{~cm}$, normal head circumference; a head circumference more than $33 \mathrm{~cm}$ and less than $37 \mathrm{~cm} \mathrm{[21].}$

We also obtained anthropometric data (height and weight), demographic data (occupation and education), and clinical data (delivery status, IUGR and history of preterm birth, abortion, delivery status, and stillbirth) of the pregnant women. To assess participants' information regarding age and gender, marital status, and educational level, we used a standard self-reported questionnaire. Subjects' weight was measured using a balanced digital scale to the nearest $100 \mathrm{~g}$, in light clothing and barefoot. Height was measured with a tape measure while the subjects were in a standing position. BMI, defined as weight in kilograms divided by height in meters squared, was calculated. General Practice Physical Activity Questionnaire (GPPAQ) was used to assess the physical activity of participants [22]. According to GPPAQ, participants were categorized into 4 levels of physical activity based on hours/week.

2.3. Statistical Analysis. As was mentioned, principal component analysis was used to identify major dietary patterns based on the 33 food groups. Three factors were considered major dietary patterns. 
TABLE 1: Food grouping used in the dietary patterns in early pregnancy.

\begin{tabular}{|c|c|}
\hline Food groups & Food items \\
\hline Bulky vegetables & Eggplant- Cabbage- Turnip- Mushroom- Squash- Stewed Pumpkin \\
\hline Leafy vegetable & Raw Vegetables- Cooked Vegetables- Celery- Spinach- Lettuce \\
\hline Colored vegetables & Tomatoes- Carrot- Pepper- Paste- Ketchup \\
\hline Green vegetables & Green Peas- Green Beans - Cucumber \\
\hline Garlic-onion & Garlic-Onion \\
\hline Fruit & $\begin{array}{l}\text { Cantaloupe-Watermelon- Pear-Apricot-Cherry Sweat-Cherry Suier Apple-Peach- Green Tomatoes- Pomegranate- } \\
\text { Plum- Bananas }\end{array}$ \\
\hline Sweat-fruit & Melon- Persimmons- Date- Fig- Grapes- Raisins- Berries- Tab and Sheet \\
\hline Citrus & Kiwi-Orange-Tangerine-Lemon and Sour Lemon-Strawberry \\
\hline Olive & Olive-Olive Oils \\
\hline Nuts & Peanuts-Almonds-Pistachios-Hazelnuts-Roasted Seeds-Walnuts \\
\hline Legumes & Beans-Peas-Lima Beans-Broad Beans-Lentils-Soy \\
\hline Saturated fat & Cream-Head-Butter-Animal Oil-Mayonnaise-Solid Oil \\
\hline Unsaturated fat & Liquid Oil - Olive Oil \\
\hline Low fat dairy & Yogurt-Whey-Cheese-Dough- Low-Fat Milk \\
\hline High fat dairy & Full Fat Milk- Chocolate Milk- Ice Cream \\
\hline Whole grain & Dark Breads (Iranian Bread)-Barley Bread -Wheat Germ-Bulgur- Barley \\
\hline Refined grains & White Bread (Lavash, Baguettes)-Rice-Toasted Bread-Sweet Bread White Flour-Biscuits-Corn- Macaroni- Noodle \\
\hline Potato & Fries -Potato \\
\hline Red meat & All Kinds of Meat- Mince Meat \\
\hline Fish & Fish-Tuna \\
\hline Poultry & Chicken with Or without Skin \\
\hline Eggs & Eggs \\
\hline Fruit juice & Fruit Juice- Lemon Juice- Compote \\
\hline Soft drink & Soft Drinks \\
\hline Sweets and dessert & Sweets- Gaz- Sohan- Chocolate- Halva \\
\hline Marinades & Pickles- Salty \\
\hline Sugar & Sugar - Candies - Sugar Cube - Honey- Jam \\
\hline Salt & Salt \\
\hline Spices & Spices \\
\hline Pizza & Pizza \\
\hline Coffee & Coffee \\
\hline Tea & Tea \\
\hline Processed meat & Sausages \\
\hline
\end{tabular}

Factor scores of dietary patterns were calculated by summing intakes of foods weighed by their factor loading for each participant. Participants were categorized by quintiles of dietary pattern scores.

Continuous variables were evaluated across quintile categories of dietary pattern scores by one-way analysis of variance. Chi-square test was used to examine significant differences in the distribution of study participants in terms of categorical variables across different quintile categories of dietary pattern scores. To investigate the association between dietary patterns and neonatal anthropometric measurements, we used multivariable logistic regression models controlled for energy intake, age, and BMI. Further adjustments were made for physical activity and social-economic levels in model 2, and additionally adjustment for delivery status, IUGR, preterm delivery, history of abortion, and stillbirth were made in model 3. All statistical tests were twosided, and $\mathrm{P}<0.05$ was considered as statistically significant. SPSS 16.0 (SPSS, Inc., Chicago, IL, USA) was used for all statistical analyses.

\section{Results}

3.1. Identified Major Dietary Patterns. Three major dietary patterns were identified according to the results obtained from the factor loading matrix (Table 2): (i) "western dietary pattern" which was greatly loaded by processed meats, fruit, fruit juice, citrus, nuts, fish, desserts and sweets, sugar, saturated fat, sweat fruit, potato, legumes, coffee, egg, pizza, 
TABLE 2: Factor loading matrix for major dietary patterns.

\begin{tabular}{lccc}
\hline Foods & \multicolumn{3}{c}{ Dietary patterns } \\
& Healthy & Western & Traditional \\
\hline Green vegetables & 0.738 & - & - \\
Leafy vegetables & 0.708 & - & - \\
Colored vegetables & 0.652 & - & 0.207 \\
Fruit & 0.598 & 0.340 & - \\
Dairy low fat & 0.591 & - & - \\
Poultry & 0.565 & - & - \\
Bulky vegetables & 0.555 & - & - \\
Red meat & 0.438 & - & - \\
Citrus & 0.428 & 0.348 & - \\
Nuts & 0.396 & 0.395 & - \\
Fish & 0.320 & 0.317 & - \\
Olive & 0.301 & - & - \\
Marinades & 0.241 & - & - \\
Fruit Juice & - & 0.650 & - \\
Sweets and dessert & - & 0.614 & - \\
Sugar & - & 0.612 & 0.271 \\
Saturated fat & - & 0.558 & - \\
Sweat fruit & 0.363 & 0.496 & - \\
Potato & - & 0.449 & - \\
Legumes & - & 0.447 & - \\
Coffee & & 0.425 & - \\
Egg & & 0.380 & - \\
Pizza & & 0.349 & - \\
High Fat Dairy & - & 0.334 & - \\
Soft Drink & & 0.328 & - \\
Whole Grain & & 0.287 & - \\
Processed Meat & & & \\
Salt & & & - \\
Spices & & & - \\
Unsaturated fat & & & - \\
Garlic onion & & & - \\
Rea & & & - \\
\hline
\end{tabular}

high fat dairy, whole grain, and soft drink; (ii) "traditional dietary pattern" which was high in refined grains, colored vegetables, olive, sugar, salt, spices, unsaturated fat, garlic, onion, and tea; (iii) "healthy dietary pattern" which was high in green vegetables, leafy vegetable, colored vegetables, fruit, low-fat dairy, poultry, bulky vegetables, red meat, citrus, nuts, fish, evil, marinades, sweat fruit, egg, and unsaturated fat.

3.2. General Characteristics and Dietary Intakes of Study Participants. According to inclusion and exclusion criteria, 812 subjects with mean (SD) maternal age of 29.4 (4.85) remained for the current analysis. Energy and nutrients intake of the study participants across different categories of healthy, western, and traditional dietary patterns were reported in Tables 3, 4, and 5, respectively. In all dietary patterns, the most of energy and nutrients intake were different in all levels of dietary patterns adherence.
The overall characteristics of the study population across different categories of dietary patterns are presented in Table 6. Women in the highest quartile of healthy dietary pattern were more likely to be employed and graduated and have employed husband, history of IUGR, and less history of early delivery compared with those in the lowest quartile. In addition, regarding the distribution of the women across categories of western dietary pattern, it was found that those with the highest adherence had significantly higher socialeconomic level, employed husband, history of cesarean, and less IUGR and stillbirth history. Furthermore, women with highest quartile of traditional dietary pattern had significantly higher social-economic level, education, and husband's education compared with those in the lowest quartile.

Data in Table 7 represent the crude and adjusted odds ratio (OR) and 95\% confidence interval (CI) for ORs from the multivariate analysis, where neonatal anthropometric measurements are the dependent variables and major maternal dietary patterns the independent variables. In all fitted models, the low level of each dietary pattern (quartile 1) was defined as the reference category. The comparison of neonatal height in different categories of maternal dietary patterns showed maternal dietary patterns were not related to neonatal height in all crude and adjusted models. Women in the highest quartile of adherence to traditional dietary pattern were $20 \%$ less likely to have short height infant compared with those in the lowest quartile [OR 0.80, 95\% (CI) (0.34-1.85), P $<0.93$ ] in the final adjusted model. Also, there were positive nonsignificant relationships between adherence to western [OR 1.31, 95\% (CI) $(0.68-2.51), \mathrm{P}=0.28$ ] and healthy [OR $1.02,95 \%(\mathrm{CI})(0.39-2.63), \mathrm{P}=0.40$ ] dietary pattern of mothers with having short height infant in the final adjusted model.

Finding about neonatal weight showed that those in the top quartile of adherence to western dietary pattern had marginally significant chance of having low weight infant compared with those in the bottom quartile in the crude model [OR 1.84, 95\% (CI) (0.94-3.60), P = 0.05]. The same results were obtained after adjusting for potential confounding variables in model 1 [OR 2.05, 95\% (CI) (1.01-4.15), $\mathrm{P}$ $=0.05$ ] and model 2 [OR 2.04, 95\% (CI) (0.97-4.32), $\mathrm{P}=$ 0.06]. After adjusting for obstetrical factors (model 3), there was a positive significant relationship between adherence to western dietary pattern [OR 5.51, 95\% (CI) (1.82-16.66), P = 0.001] with having low birth weight infant.

In addition, lower marginal significant chance of having low weight infant was found among women who were in the top adherence of traditional dietary pattern [OR 0.76 , 95\% (CI) (0.32-1.77), P = 0.05] in the crude model. Although this association became significant even after taking potential confounders into account in model 1 [OR 0.80, 95\% (CI) (0.34-1.93), $\mathrm{P}<0.01$ ], such association was not seen in models 2 and 3. However, maternal adherence to healthy dietary pattern was not related to birth weight of infants in all crude and adjusted models.

We found no significant association either between maternal western dietary pattern and head circumference of infant or between healthy or traditional dietary pattern and mentioned measure. In final adjusted model, women with a higher adherence to western dietary pattern had 4.88 times 
TABLE 3: Energy and nutrients intake of the study participants across different categories of healthy dietary pattern score.

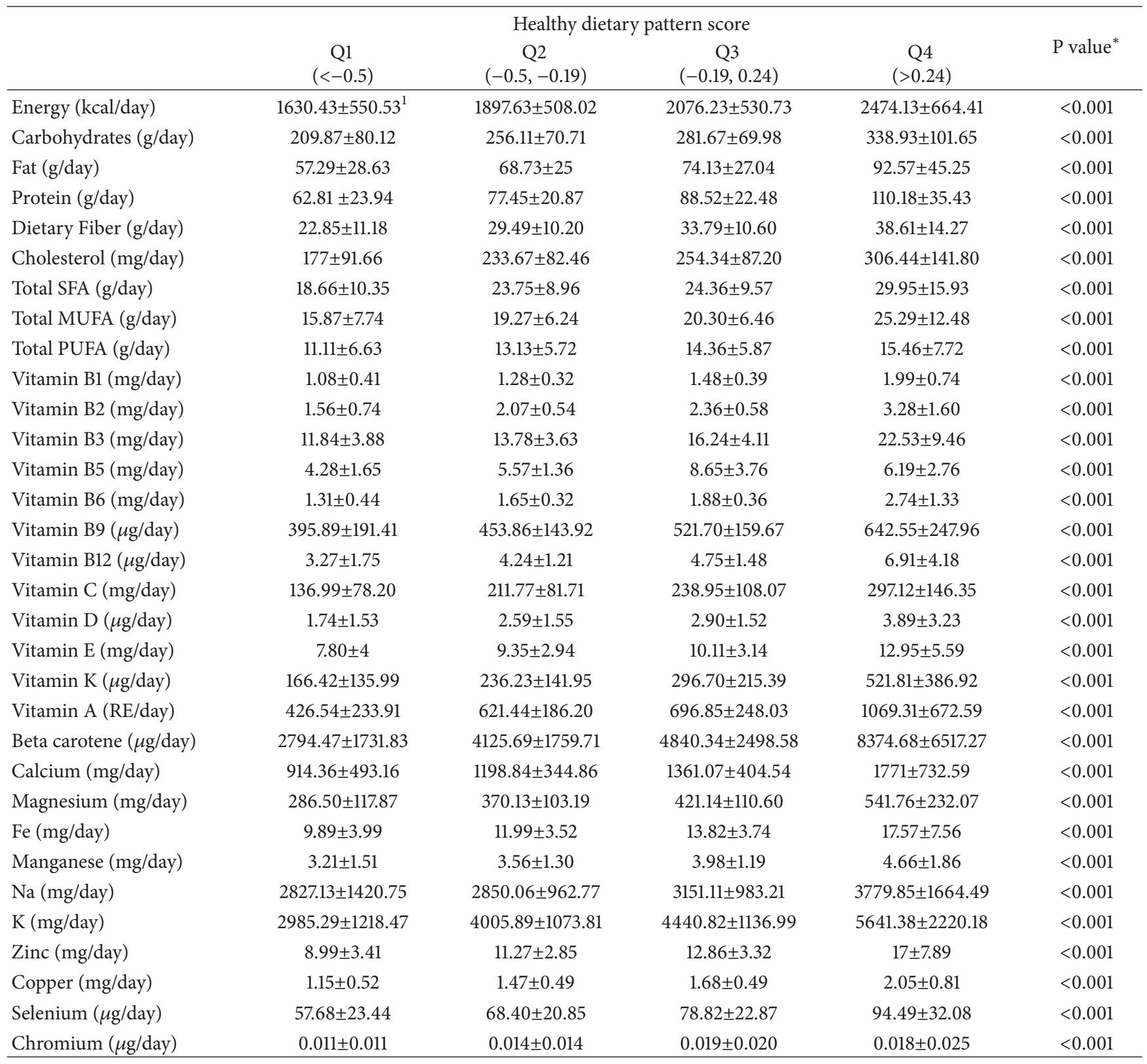

MUFA: monounsaturated fatty acid; PUFA: polyunsaturated fatty acid; SFA: saturated fatty acid.

* Resulted from analysis of variance.

${ }^{1}$ Values are presented as mean \pm SD.

larger odds for having an infant with low birth head circumference than those with a lower adherence level (OR: 4.88; $95 \%$ CI: 0.79-30.19 P = 0.35). Same result was obtained about healthy dietary pattern [OR 3.70, 95\% (CI) (0.72-17.86), P = $0.52]$.

Furthermore, lower risk of low birth head circumference was nonsignificantly associated with a traditional dietary pattern [OR 0.58, 95\% (CI) (0.13-2.51), P = 0.41].

\section{Discussion}

Current study on 812 subjects is one of the largest studies evaluating the association between major maternal dietary patterns during the first-trimester period of pregnancy and neonatal anthropometric measurements. This study demonstrated a significant association between high adherences to western dietary pattern and chance of having low birth weight infant.

However, such associations were not seen with healthy and traditional dietary pattern. Earlier studies have mostly examined the effects of particular nutrients or foods during gestational period on neonatal anthropometric measurements [13] and few data are available linking habitual diet to neonatal anthropometric measurements.

Western dietary pattern as an unhealthy food choice is unable to fulfill the increased key micronutrients requirements 
TABLE 4: Energy and nutrients intake of the study participants across different categories of western dietary pattern score.

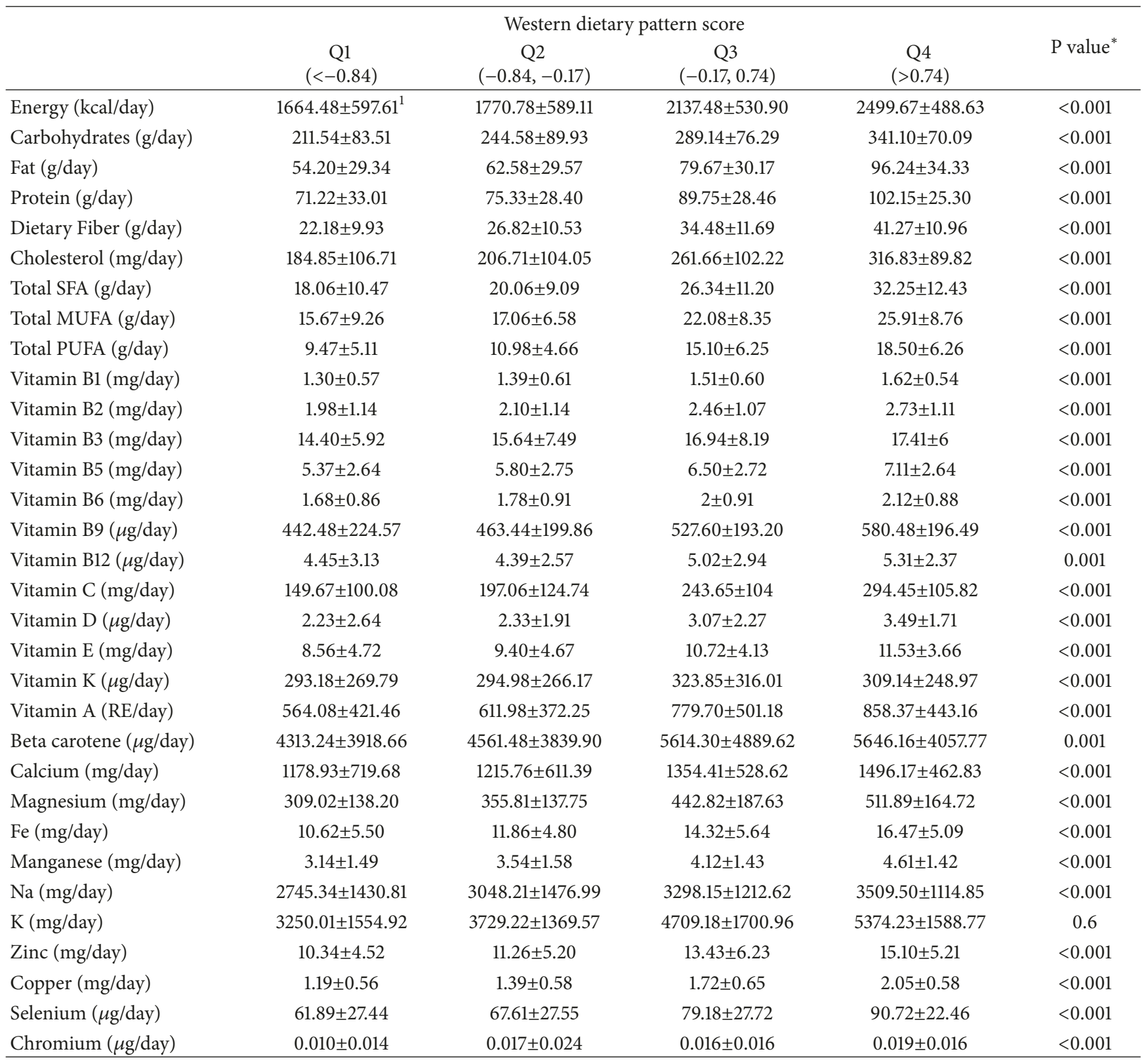

MUFA: monounsaturated fatty acid; PUFA: polyunsaturated fatty acid; SFA: saturated fatty acid.

* Resulted from analysis of variance.

${ }^{1}$ Values are presented as mean \pm SD.

for normal fetal growth during pregnancy and results in such adverse effects on the fetus [23]. Earlier studies have also shown that high-quality diet assessed by using the Alternate Healthy Eating Index (AHEI) in the first trimester of pregnancy is positively associated with birth size including birth weight and birth length [15]. Other publication has also reported a protective effect of adherence to Mediterranean diet during pregnancy and risk of delivering a fetal growth-restricted infant for weight in Atlantic area [16].

In a population-based prospective study, it has been shown that women in the "wheat products" pattern had higher odds of having infants with the low birth weight and head circumference compared with women in the "rice, fish, and vegetables" pattern [24]. Also, some maternal dietary patterns characterized by high intakes of vegetable, fruit, white rice [25], Vitamin C, milk, and fat [26] are associated with larger birth size of infant. In contrast maternal intakes of carbohydrate were negatively associated with infant length and abdominal circumference [26].

Present study showed that the women with prior problematic pregnancies or higher socioeconomic status or education level were more prone to have a healthy dietary pattern. These results were in line with Northstone et al. study which showed that "health conscious" dietary patterns were 
TABLE 5: Energy and nutrients intake of the study participants across different categories of traditional dietary pattern score.

\begin{tabular}{|c|c|c|c|c|c|}
\hline & & Traditional die & pattern score & & \\
\hline & $\begin{array}{c}\text { Q1 } \\
(<-0.48)\end{array}$ & $\begin{array}{c}\mathrm{Q} 2 \\
(-0.48,-0.12) \\
\end{array}$ & $\begin{array}{c}\text { Q3 } \\
(-0.12,0.32) \\
\end{array}$ & $\begin{array}{c}\mathrm{Q} 4 \\
(>0.32) \\
\end{array}$ & P value* \\
\hline Energy (kcal/day) & $1801.65 \pm 650.43$ & $2004.22 \pm 592.22$ & $2052.10 \pm 560.61$ & $2230.27 \pm 688.64$ & $<0.001$ \\
\hline Carbohydrates (g/day) & $244.24 \pm 105.66$ & $261.87 \pm 74.35$ & $275.25 \pm 75.01$ & $305.01 \pm 105.05$ & $<0.001$ \\
\hline Fat (g/day) & $62.37 \pm 33.35$ & $72.72 \pm 32.67$ & $75.02 \pm 31.65$ & $82.66 \pm 38.52$ & $<0.001$ \\
\hline Protein (g/day) & $78.73 \pm 36.18$ & $82.83 \pm 28.07$ & $85.98 \pm 27.29$ & $90.92 \pm 32.11$ & 0.001 \\
\hline Dietary Fiber (g/day) & $28.72 \pm 14.40$ & $31.44 \pm 12.13$ & $31.62 \pm 11$ & $32.97 \pm 13.96$ & $<0.001$ \\
\hline Cholesterol (mg/day) & $220.63 \pm 125.12$ & $242.19 \pm 98.82$ & $252.27 \pm 100.89$ & $255.10 \pm 122.42$ & $<0.001$ \\
\hline Total SFA (g/day) & $21.68 \pm 13.98$ & $24.18 \pm 11.29$ & $24.73 \pm 10.60$ & $26.16 \pm 12.32$ & $<0.001$ \\
\hline Total MUFA (g/day) & $17.62 \pm 9.67$ & $19.39 \pm 8.75$ & $20.14 \pm 6.77$ & $23.59 \pm 10.37$ & $<0.001$ \\
\hline Total PUFA (g/day) & $11.70 \pm 6.86$ & $13.69 \pm 6.37$ & $13.37 \pm 5.52$ & $15.31 \pm 7.51$ & $<0.001$ \\
\hline Vitamin B1 (mg/day) & $1.32 \pm 0.66$ & $1.38 \pm 0.46$ & $1.46 \pm 0.46$ & $1.67 \pm 0.70$ & $<0.001$ \\
\hline Vitamin B2 (mg/day) & $2.09 \pm 1.24$ & $2.19 \pm 0.77$ & $2.39 \pm 1.11$ & $2.60 \pm 1.34$ & $<0.001$ \\
\hline Vitamin B3 (mg/day) & $15.25 \pm 7.73$ & $15.05 \pm 5.91$ & $16.09 \pm 6.19$ & $18 \pm 7.82$ & $<0.001$ \\
\hline Vitamin B5 (mg/day) & $5.65 \pm 3.07$ & $5.84 \pm 1.88$ & $6.28 \pm 2.52$ & $7.01 \pm 3.21$ & $<0.001$ \\
\hline Vitamin B6 (mg/day) & $1.75 \pm 0.97$ & $1.80 \pm 0.78$ & $1.91 \pm 0.79$ & $2.13 \pm 1.01$ & $<0.001$ \\
\hline Vitamin B9 ( $\mu \mathrm{g} /$ day) & $440.44 \pm 220.02$ & $489.13 \pm 171.98$ & $506.64 \pm 162.56$ & $577.79 \pm 253.04$ & $<0.001$ \\
\hline Vitamin B12 ( $\mu \mathrm{g} /$ day) & $4.36 \pm 3.18$ & $4.54 \pm 2.33$ & $4.92 \pm 2.42$ & $5.35 \pm 3.06$ & 0.002 \\
\hline Vitamin C (mg/day) & $201.36 \pm 123.75$ & $224.14 \pm 114.97$ & $222.56 \pm 98.81$ & $236.77 \pm 142.37$ & 0.03 \\
\hline Vitamin D ( $\mu \mathrm{g} /$ day $)$ & $2.57 \pm 2.53$ & $2.77 \pm 1.72$ & $2.94 \pm 2.18$ & $2.83 \pm 2.38$ & 0.4 \\
\hline Vitamin E (mg/day) & $7.97 \pm 4.19$ & $9.34 \pm 3.43$ & $10.02 \pm 3.42$ & $12.89 \pm 5.08$ & $<0.001$ \\
\hline Vitamin K ( $\mu \mathrm{g} /$ day) & $300.64 \pm 303.55$ & $278.69 \pm 229.28$ & $316.65 \pm 280.08$ & $325.17 \pm 285.80$ & $<0.001$ \\
\hline Vitamin A (RE/day) & $662.33 \pm 571.37$ & $677.87 \pm 343.67$ & $729.23 \pm 409.11$ & $744.70 \pm 452.74$ & 0.2 \\
\hline Beta carotene ( $\mu \mathrm{g} /$ day $)$ & $4928.33 \pm 5105.30$ & $4680.14 \pm 3613.57$ & $5083.92 \pm 3937.47$ & $5442.80 \pm 4123.45$ & 0.3 \\
\hline Calcium (mg/day) & $1155.88 \pm 631.37$ & $1267.82 \pm 451.51$ & $1370.70 \pm 588.89$ & $1450.87 \pm 670.68$ & $<0.001$ \\
\hline Magnesium (mg/day) & $375.19 \pm 221.24$ & $398.22 \pm 140.64$ & $408.83 \pm 142.54$ & $437.29 \pm 184.32$ & 0.005 \\
\hline $\mathrm{Fe}$ (mg/day) & $12.30 \pm 6.40$ & $13.14 \pm 5.61$ & $13.18 \pm 4.26$ & $14.65 \pm 6.15$ & $<0.001$ \\
\hline Manganese (mg/day) & $3.17 \pm 1.65$ & $3.76 \pm 1.27$ & $3.93 \pm 1.26$ & $4.55 \pm 1.77$ & $<0.001$ \\
\hline $\mathrm{Na}$ (mg/day) & $2220.44 \pm 1055.73$ & $3029.23 \pm 937.70$ & $3125.09 \pm 934.55$ & $4237.76 \pm 1529.59$ & \\
\hline $\mathrm{K}$ (mg/day) & $3871.97 \pm 2067.89$ & $4193.12 \pm 1465.80$ & $4318.04 \pm 1365.94$ & $4678.99 \pm 1960.44$ & 0.3 \\
\hline Zinc (mg/day) & $11.69 \pm 6.87$ & $12.06 \pm 4.13$ & $12.61 \pm 4.96$ & $13.77 \pm 6$ & 0.001 \\
\hline Copper (mg/day) & $1.50 \pm 0.80$ & $1.58 \pm 0.62$ & $1.58 \pm 0.57$ & $1.70 \pm 0.67$ & 0.02 \\
\hline Selenium ( $\mu \mathrm{g} /$ day) & $66.58 \pm 31.15$ & $73.55 \pm 23.94$ & $75.70 \pm 23.97$ & $83.56 \pm 31.82$ & $<0.001$ \\
\hline Chromium ( $\mu$ g/day) & $0.012 \pm 0.018$ & $0.016 \pm 0.015$ & $0.015 \pm 0.016$ & $0.01 \pm 0.02$ & 0.004 \\
\hline
\end{tabular}

MUFA: monounsaturated fatty acid; PUFA: polyunsaturated fatty acid; SFA: saturated fatty acid.

* Resulted from analysis of variance.

${ }^{1}$ Values are presented as mean \pm SD.

negatively associated with decreasing educational level, age, and socioeconomic status [21]. Also, dietary surveys during pregnancy have suggested the poor diet quality and quantity of low socioeconomic groups [27]. It can be concluded that women with low socioeconomic status need specific dietary intervention programs due to increase in nutritional demands during pregnancy.

As was mentioned, we did not see any associations of healthy and traditional dietary patterns with consumption of red meats in the healthy dietary pattern and refined grain, salt, and sugar in the traditional dietary pattern. Mentioned foods as an unhealthy food might have been given an adverse score in the healthy and traditional dietary patterns in the current study. This might be a reason why we failed to report a significant positive association between high adherence to healthy dietary pattern and neonatal anthropometric measurements.

It should be mentioned that refined grains are major source of carbohydrate intake in Iranian diet, which has been shown to be associated with higher risk of some chronic disorders. Iranian usual diet also contains high amounts of salt, which means that salt intake among them is almost twofold the recommended levels. In addition, fruits, vegetables, and low-fat dairy intake among them is low; however, legumes 


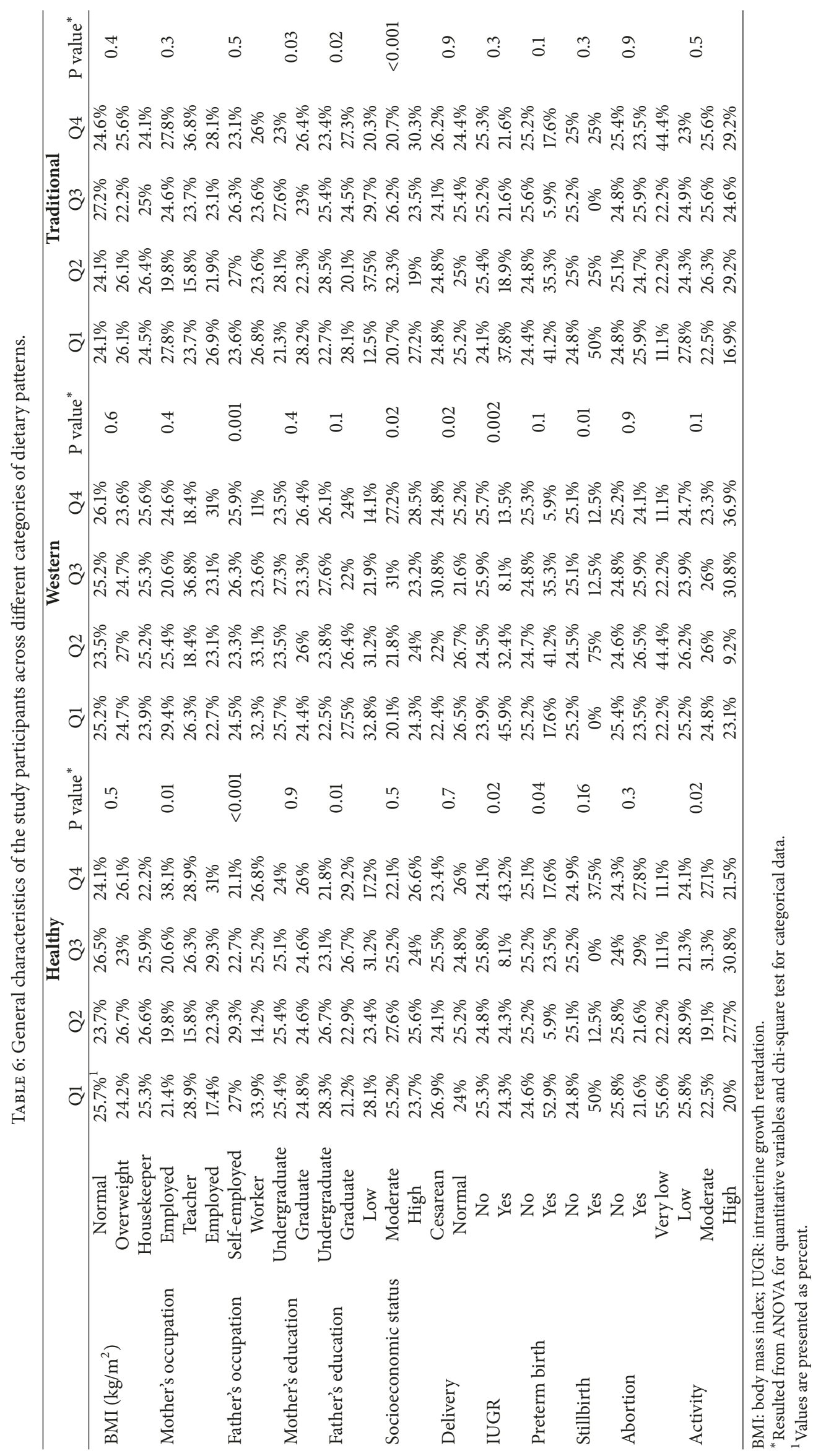




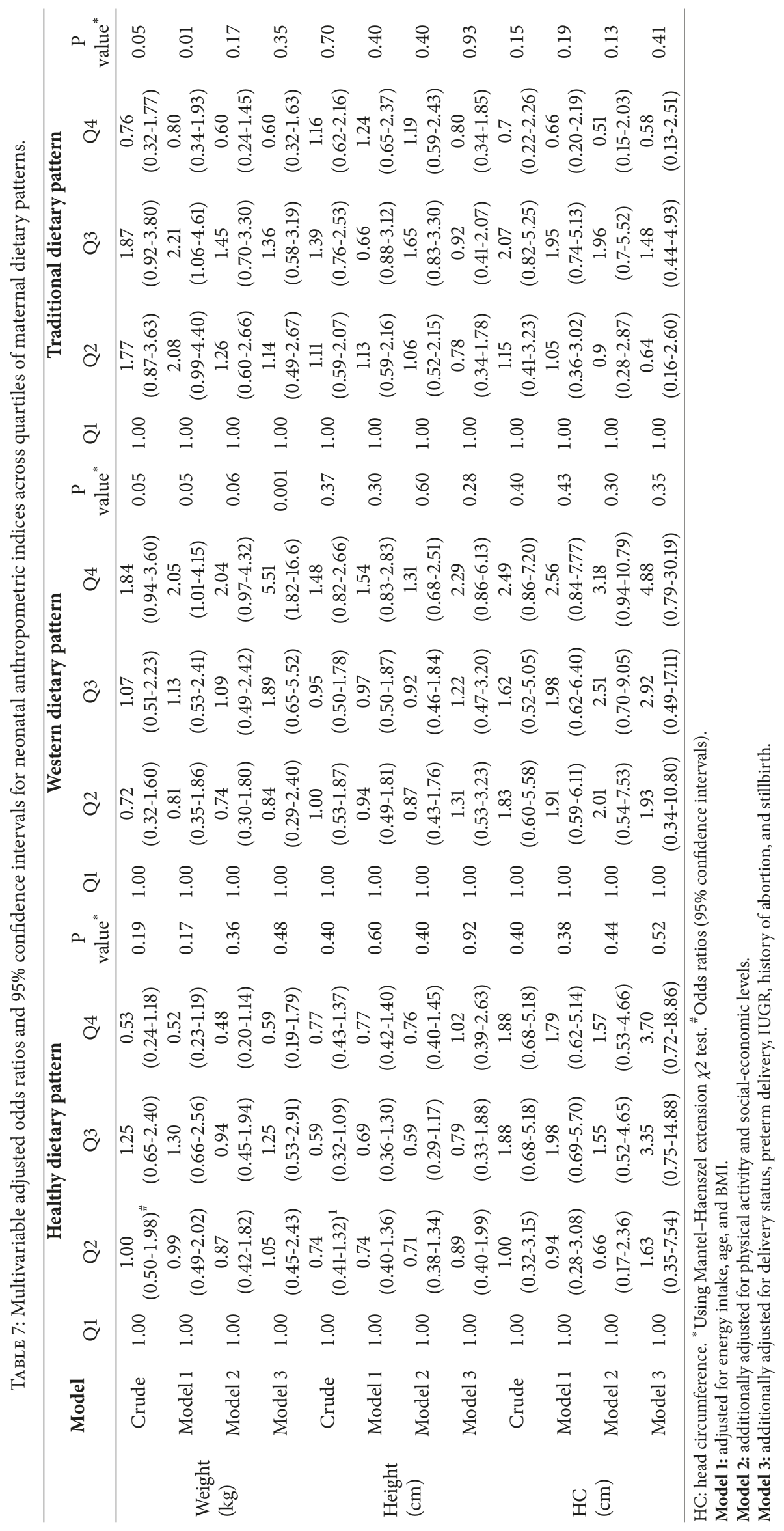


intake is good in the mentioned population [28]. Therefore, understanding the differences between healthy, western, and traditional dietary patterns and usual Iranian dietary pattern might help interpreting the importance of the findings we reached.

Also, postpregnancy dietary habits and choices may be different compared with prepregnancy. The majority of pregnant women are encouraged to healthier dietary changes. We cannot overlook that the long time adherence to a specific diet affects health outcomes.

Another point that needs to be considered is the measurements error in nutritional assessment; this may increase the likelihood of misclassification, contributing to the discrepancy among our results.

Detailed information about food preparation and brands is also lacking in this instrument.

Another potential drawback should be also considered. Lack of funding to obtain fetal weights estimated via serial ultrasound is an issue in the analyses. It has been suggested that intrauterine growth patterns improved prediction of childhood anthropometry, above and beyond birth weight alone. Moreover, up to $70 \%$ of small-for-gestational-age (SGA) infants are constitutionally small ("small but healthy"), which make SGA birth a poor surrogate for intrauterine growth restriction [29]. In this regard, intrauterine growth should be identified on the basis of measures of fetal growth patterns, rather than birth weight [30].

In addition, residual confounding due to genetic backgrounds could not be excluded, although we controlled some important potential confounding factors. Therefore, these potential limitations should be considered in the interpretation of our results. However, the main strengths of the current study include its multidisciplinary scope including epidemiology, statistics, pediatrics, maternity, and nutrition. Another positive point is that few studies have collected data on maternal dietary patterns and neonatal anthropometric measurements.

In summary, evaluations carried out in the present study indicate that during early pregnancy mothers of low birth weight infant had a different dietary pattern and more adhered to the western dietary pattern compared to mothers of normal weight birth infant. Therefore, intrauterine growth conditions might be improved through the adoption of certain dietary patterns during pregnancy. Larger studies are needed to determine whether maternal dietary patterns lead to significant modifications in neonatal anthropometric measurements.

\section{Conflicts of Interest}

The authors have no conflicts of interest.

\section{Acknowledgments}

Food Security Research Center of Isfahan University of Medical Sciences supported this study.

\section{References}

[1] Y.-Q. Zhang and H. Li, "Changes in weight, length, head circumference, and ponderal index at birth of healthy term newborns in nine cities in China during the period of rapid social development 1985-2005," Economics \& Human Biology, vol. 19, pp. 45-50, 2015.

[2] Z. Iheozor-Ejiofor, P. Middleton, M. Esposito, and A.-M. Glenny, "Treating periodontal disease for preventing adverse birth outcomes in pregnant women," Cochrane Database of Systematic Reviews, no. 6, Article ID CD005297, 2017.

[3] H. Long, J.-M. Yi, P.-L. Hu et al., "Benefits of Iron supplementation for low birth weight infants: a systematic review," $B M C$ Pediatrics, vol. 12, article 99, 2012.

[4] M. Sicard, S. Nusinovici, M. Hanf et al., "Fetal and postnatal head circumference growth: Synergetic factors for neurodevelopmental outcome at 2 years of age for preterm infants," Neonatology, vol. 112, no. 2, pp. 122-129, 2017.

[5] S. Kuhle, B. Maguire, N. Ata, N. MacInnis, and L. Dodds, "Birth weight for gestational age, anthropometric measures, and cardiovascular disease markers in children," The Journal of Pediatrics, vol. 182, pp. 99-106, 2017.

[6] S. Visentin, F. Grumolato, G. B. Nardelli, B. Di Camillo, E. Grisan, and E. Cosmi, "Early origins of adult disease: low birth weight and vascular remodeling," Atherosclerosis, vol. 237, no. 2, pp. 391-399, 2014.

[7] T. L. Pham, J. He, A. H. Kakazu, B. Jun, N. G. Bazan, and H. E. P. Bazan, "Defining a mechanistic link between pigment epithelium-derived factor, docosahexaenoic acid, and corneal nerve regeneration," The Journal of Biological Chemistry, vol. 292, no. 45, pp. 18486-18499, 2017.

[8] D. Hirano, K. Ishikura, O. Uemura et al., "Association between low birth weight and childhood-onset chronic kidney disease in Japan: A combined analysis of a nationwide survey for paediatric chronic kidney disease and the National Vital Statistics Report," Nephrology Dialysis Transplantation, vol. 31, no. 11, pp. 1895-1900, 2016.

[9] P. Chen, A. Chu, W. Liao et al., "Prenatal growth patterns and birthweight are associated with differential DNA methylation and gene expression of cardiometabolic risk genes in human placentas: a discovery-based Approach," Reproductive Sciences, vol. 25, no. 4, pp. 523-539, 2017.

[10] A. N. Sferruzzi-Perri, I. Sandovici, M. Constancia, and A. L. Fowden, "Placental phenotype and the insulin-like growth factors: resource allocation to fetal growth," The Journal of Physiology, vol. 595, no. 15, pp. 5057-5093, 2017.

[11] S. V. Wrottesley, C. Lamper, and P. T. Pisa, "Review of the importance of nutrition during the first 1000 days: maternal nutritional status and its associations with fetal growth and birth, neonatal and infant outcomes among African women," Journal of Developmental Origins of Health and Disease, vol. 7, no. 2, pp. 144-162, 2016.

[12] W. H. Seo, B. M. Choi, H. Lee, Y. Yoo, J. T. Choung, and Y. Han, "Effect of the prenatal maternal environments and diets on cord blood interleukin-4 and interferon -gamma: a pilot study," Asian Pacific Journal of Allergy and Immunology, vol. 35, no. 1, pp. 46-53, 2017.

[13] M. M. Murphy, N. Stettler, K. M. Smith, and R. Reiss, "Associations of consumption of fruits and vegetables during pregnancy with infant birth weight or small for gestational age births: A systematic review of the literature," International Journal of Women's Health, vol. 6, pp. 899-912, 2014.

[14] S. Naghavi, S. B. Mahdavi, B. Moradi, M. H. Tajadini, and R. Kelishadi, "Association of dietary patterns during pregnancy and cord blood nitric oxide level with birth weight of newborns," International Journal of Pediatrics, vol. 5, no. 3, pp. 4489-4501, 2017. 
[15] C. L. Rodríguez-Bernal, M. Rebagliato, C. Iñiguez et al., "Diet quality in early pregnancy and its effects on fetal growth outcomes: The infancia y medio ambiente (childhood and environment) mother and child cohort study in Spain," American Journal of Clinical Nutrition, vol. 91, no. 6, pp. 1659-1666, 2010.

[16] L. Chatzi, M. Mendez, R. Garcia et al., "Mediterranean diet adherence during pregnancy and fetal growth: INMA (Spain) and RHEA (Greece) mother-child cohort studies," British Journal of Nutrition, vol. 107, no. 1, pp. 135-145, 2012.

[17] F. B. Hu, "Dietary pattern analysis: a new direction in nutritional epidemiology," Current Opinion in Lipidology, vol. 13, no. 1, pp. 3-9, 2002.

[18] A. K. Kant, B. I. Graubard, and A. Schatzkin, "Dietary patterns predict mortality in a national cohort: the National Health Interview Surveys, 1987 and 1992," Journal of Nutrition, vol. 134, no. 7, pp. 1793-1799, 2004.

[19] S. Rafieifar, H. Pouraram, A. Djazayery et al., "Strategies and opportunities ahead to reduce salt intake," Archives of Iranian Medicine, vol. 19, no. 10, pp. 729-734, 2016.

[20] R. Hashemi, A. D. Motlagh, R. Heshmat et al., "Diet and its relationship to sarcopenia in community dwelling iranian elderly: A cross sectional study," Nutrition Journal, vol. 31, no. 1, pp. 97-104, 2015.

[21] K. Northstone, P. Emmett, and I. Rogers, "Dietary patterns in pregnancy and associations with socio-demographic and lifestyle factors," European Journal of Clinical Nutrition, vol. 62, no. 4, pp. 471-479, 2008.

[22] For Nursing NCC, The General Practice Physical Activity Questionnaire (GPPAQ), 2008.

[23] V. K. Knudsen, I. M. Orozova-Bekkevold, T. B. Mikkelsen, S. Wolff, and S. F. Olsen, "Major dietary patterns in pregnancy and fetal growth," European Journal of Clinical Nutrition, vol. 62, no. 4, pp. 463-470, 2008.

[24] H. Okubo, Y. Miyake, S. Sasaki et al., "Maternal dietary patterns in pregnancy and fetal growth in Japan: the Osaka Maternal and Child Health Study," British Journal of Nutrition, vol. 107, no. 10, pp. 1526-1533, 2012.

[25] A.-R. Chia, J. V. De Seymour, M. Colega et al., "A vegetable, fruit, and white rice dietary pattern during pregnancy is associated with a lower risk of preterm birth and larger birth size in a multiethnic Asian cohort: The Growing Up in Singapore Towards healthy Outcomes (GUSTO) cohort study," American Journal of Clinical Nutrition, vol. 104, no. 5, pp. 1416-1423, 2016.

[26] K. G. Hjertholm, P. O. Iversen, G. Holmboe-Ottesen et al., "Maternal dietary intake during pregnancy and its association to birth size in rural Malawi: A cross-sectional study," Maternal \& Child Nutrition, vol. 14, no. 1, Article ID e12433, 2018.

[27] W. Doyle, M. A. Crawford, B. M. Laurance, and P. Drury, "Dietary survey during pregnancy in a low socio-economic group," Human Nutrition: Applied Nutrition, vol. 36, no. 2, pp. 95-106, 1982.

[28] G. Valipour, A. Esmaillzadeh, L. Azadbakht, H. Afshar, A. Hassanzadeh, and P. Adibi, "Adherence to the DASH diet in relation to psychological profile of Iranian adults," European Journal of Nutrition, vol. 56, no. 1, pp. 309-320, 2017.

[29] No GtG, The investigation and management of the small-forgestational-age fetus, 2002.

[30] J. A. Hutcheon, G. W. Jacobsen, M. S. Kramer, M. Martinussen, and R. W. Platt, "Small size at birth or abnormal intrauterine growth trajectory: Which matters more for child growth?" American Journal of Epidemiology, vol. 183, no. 12, pp. 1107-1113, 2016. 


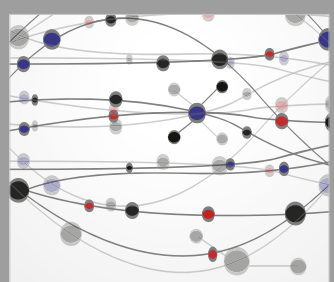

The Scientific World Journal
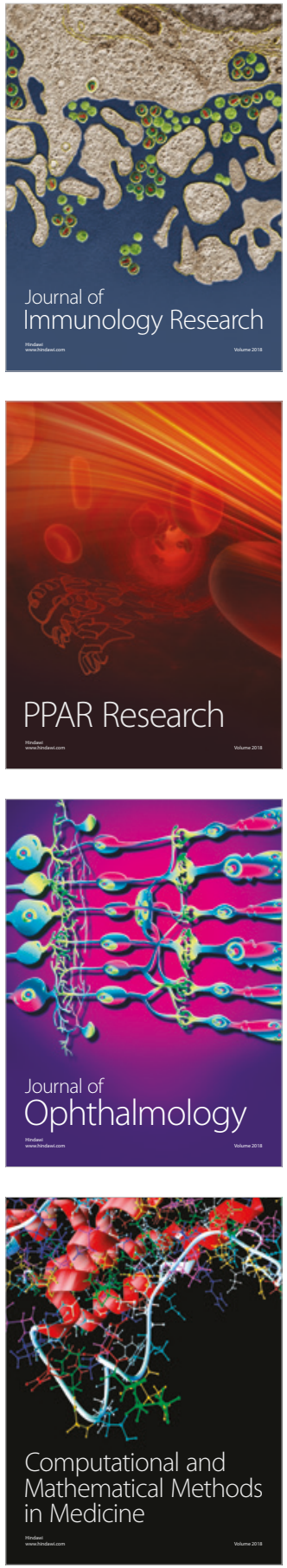

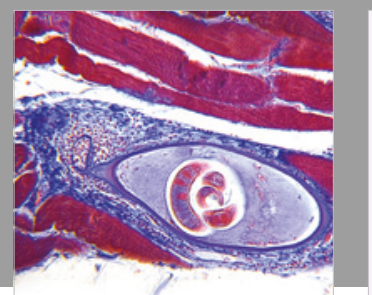

Gastroenterology Research and Practice

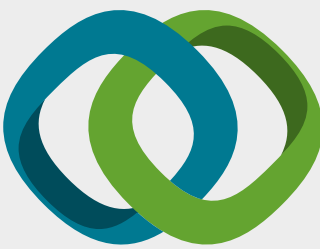

\section{Hindawi}

Submit your manuscripts at

www.hindawi.com
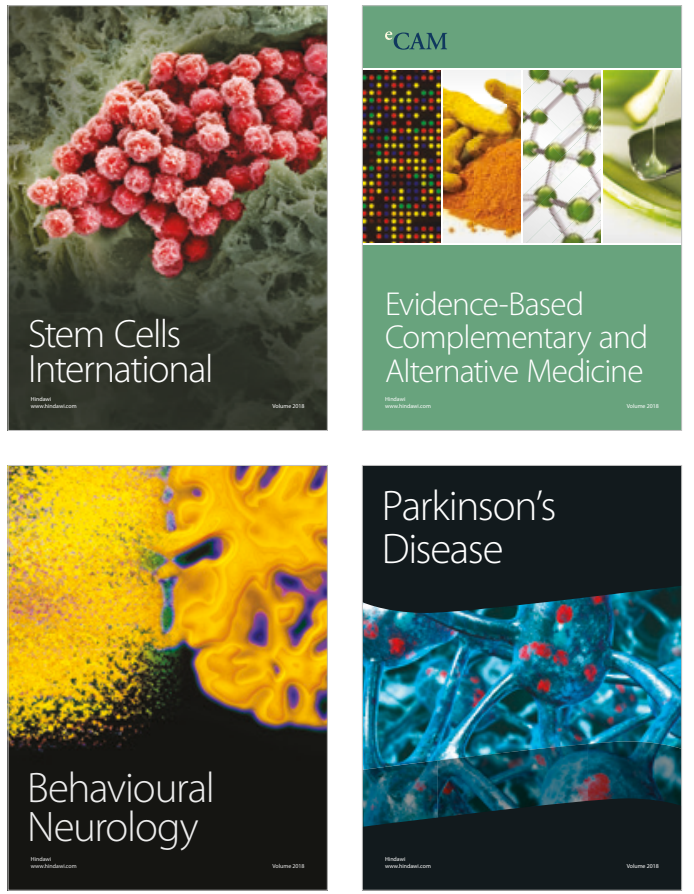

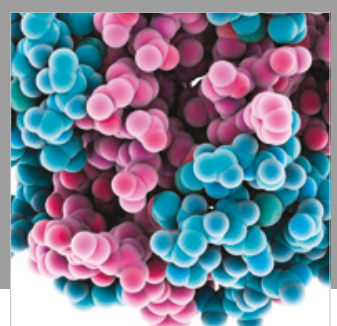

ournal of

Diabetes Research

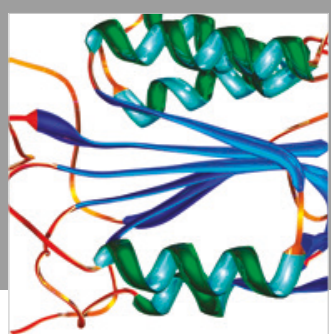

Disease Markers
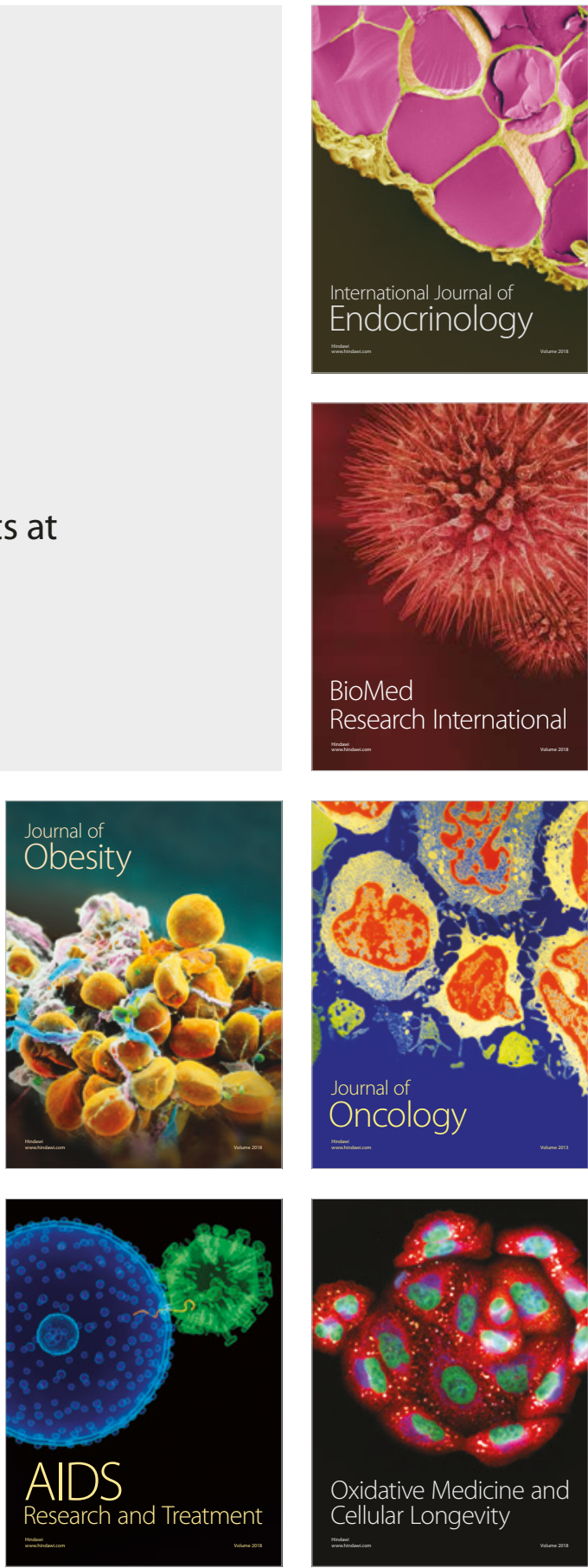